\title{
Genome wide association study of response to interval and continuous exercise training: the Predict-HIIT study
}

Camilla J. Williams ${ }^{1 \dagger}$, Zhixiu Li ${ }^{2 \dagger}$, Nicholas Harvey ${ }^{3,4+}$, Rodney A. Lea ${ }^{4}$, Brendon J. Gurd ${ }^{5}$, Jacob T. Bonafiglia ${ }^{5}$, loannis Papadimitriou ${ }^{6}$, Macsue Jacques ${ }^{6}$, Ilaria Croci ${ }^{1,7,20}$, Dorthe Stensvold ${ }^{7}$, Ulrik Wisloff ${ }^{1,7}$, Jenna L. Taylor ${ }^{1}$, Trishan Gajanand', Emily R. Cox ${ }^{1}$, Joyce S. Ramos ${ }^{1,8}$, Robert G. Fassett ${ }^{1}$, Jonathan P. Little ${ }^{9}$, Monique E. Francois ${ }^{9}$, Christopher M. Hearon Jr ${ }^{10}$, Satyam Sarma ${ }^{10}$, Sylvan L. J. E. Janssen ${ }^{10,11}$, Emeline M. Van Craenenbroeck ${ }^{12}$, Paul Beckers ${ }^{12}$, Véronique A. Cornelissen ${ }^{13}$, Erin J. Howden ${ }^{14}$, Shelley E. Keating ${ }^{1}$, Xu Yan ${ }^{6,15}$, David J. Bishop ${ }^{6,16}$, Anja Bye ${ }^{7,17}$, Larisa M. Haupt ${ }^{4}$, Lyn R. Griffiths ${ }^{4}$, Kevin J. Ashton ${ }^{3}$, Matthew A. Brown ${ }^{18}$, Luciana Torquati ${ }^{19}$, Nir Eynon ${ }^{6}$ and Jeff S. Coombes ${ }^{1 *}$

\begin{abstract}
Background: Low cardiorespiratory fitness $\left(\mathrm{VO}_{2 \text { peak }}\right)$ is highly associated with chronic disease and mortality from all causes. Whilst exercise training is recommended in health guidelines to improve $\mathrm{VO}_{2 \text { peak, }}$, there is considerable inter-individual variability in the $\mathrm{VO}_{2 \text { peak }}$ response to the same dose of exercise. Understanding how genetic factors contribute to $\mathrm{VO}_{2 \text { peak }}$ training response may improve personalisation of exercise programs. The aim of this study was to identify genetic variants that are associated with the magnitude of $\mathrm{VO}_{2}$ peak response following exercise training.

Methods: Participant change in objectively measured $\mathrm{VO}_{2}$ peak from 18 different interventions was obtained from a multi-centre study (Predict-HIIT). A genome-wide association study was completed ( $n=507)$, and a polygenic predictor score (PPS) was developed using alleles from single nucleotide polymorphisms (SNPs) significantly associated $\left(P<1 \times 10^{-5}\right)$ with the magnitude of $\mathrm{VO}_{2}$ peak response. Findings were tested in an independent validation study $(n=39)$ and compared to previous research.
\end{abstract}

Results: No variants at the genome-wide significance level were found after adjusting for key covariates (baseline $\mathrm{VO}_{2}$ peak individual study, principal components which were significantly associated with the trait). A Quantile-Quantile plot indicates there was minor inflation in the study. Twelve novel loci showed a trend of association with $\mathrm{VO}_{2}$ peak response that reached suggestive significance $\left(P<1 \times 10^{-5}\right)$. The strongest association was found near the membrane associated guanylate kinase, WW and PDZ domain containing 2 (MAG/2) gene ( $\left.r 6959961, P=2.61 \times 10^{-7}\right)$. A PPS created from the 12 lead SNPs was unable to predict $\mathrm{VO}_{2}$ peak response in a tenfold cross validation, or in an independent $(n=39)$ validation study $(P>0.1)$. Significant correlations were found for beta coefficients of variants in the Predict-HIIT

\footnotetext{
*Correspondence: jcoombes@uq.edu.au

${ }^{\dagger}$ Camilla J. Williams, Zhixiu Li and Nicholas Harvey_co first authors

${ }^{1}$ Centre for Research on Exercise, Physical Activity and Health, School

of Human Movement and Nutrition Sciences, University of Queensland,

St. Lucia, Brisbane, QLD, Australia

Full list of author information is available at the end of the article
}

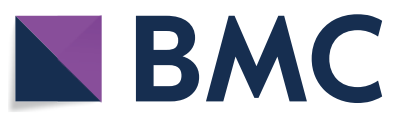

(c) The Author(s) 2021. Open Access This article is licensed under a Creative Commons Attribution 4.0 International License, which permits use, sharing, adaptation, distribution and reproduction in any medium or format, as long as you give appropriate credit to the original author(s) and the source, provide a link to the Creative Commons licence, and indicate if changes were made. The images or other third party material in this article are included in the article's Creative Commons licence, unless indicated otherwise in a credit line to the material. If material is not included in the article's Creative Commons licence and your intended use is not permitted by statutory regulation or exceeds the permitted use, you will need to obtain permission directly from the copyright holder. To view a copy of this licence, visit http://creativecommons.org/licenses/by/4.0/. The Creative Commons Public Domain Dedication waiver (http://creativeco mmons.org/publicdomain/zero/1.0/) applies to the data made available in this article, unless otherwise stated in a credit line to the data. 
$\left(P<1 \times 10^{-4}\right)$ and the validation study $\left(P<\times 10^{-6}\right)$, indicating that general effects of the loci exist, and that with a higher statistical power, more significant genetic associations may become apparent.

Conclusions: Ongoing research and validation of current and previous findings is needed to determine if genetics does play a large role in $\mathrm{VO}_{2}$ peak response variance, and whether genomic predictors for $\mathrm{VO}_{2}$ peak response trainability can inform evidence-based clinical practice.

Trial registration Australian New Zealand Clinical Trials Registry (ANZCTR), Trial Id: ACTRN12618000501246, Date Registered: 06/04/2018, http://www.anzctr.org.au/Trial/Registration/TrialReview.aspx?id=374601\&isReview=true.

Keywords: Genetics, $\mathrm{VO}_{2}$ peak training response, Individual variability, GWAS, Polygenic predictor score

\section{Background}

Cardiorespiratory fitness (CRF) is measured by peak oxygen uptake $\left(\mathrm{VO}_{2}\right.$ peak) during a graded exercise test, and is strongly associated with a reduced risk of cardiometabolic diseases and mortality [1]. Improving $\mathrm{VO}_{2}$ peak can generally be achieved by regular endurance exercise training, in a dose-dependent manner [2]. Data typically supports the notion that a higher dose of exercise (volume and intensity) will elicit greater $\mathrm{VO}_{2}$ peak gains [3-7]. Interval training, such as sprint interval training (SIT) and high-intensity interval training (HIIT) have shown comparable [8] and greater [9-13] group mean $\mathrm{VO}_{2}$ peak changes, respectively, compared with moderate-intensity continuous training (MICT). However, there is considerable inter-individual variability in observed $\mathrm{VO}_{2}$ peak improvements following apparently similar exercise training [7, 14]. Identifying the genetic and environmental determinants that can predict exercise response may pave the way to personalised exercise programs that can maximise health outcomes.

An early genome wide association study (GWAS) using data from the HEalth, RIsk factors, exercise Training And GEnetics (HERITAGE) Family Study reported that 21 variants contributed to $49 \%$ of the variance in $\mathrm{VO}_{2}$ peak response [15]. However, very few of these variants have been replicated in further testing or other studies suggesting that the variants identified in the HERITAGE study were overfitted to the specific population. In a recent systematic review, we identified 35 studies describing 15 cohorts that found 97 possible variants associated with $\mathrm{VO}_{2}$ peak training response [16]. Only 13 genetic variants were replicated by more than two authors $[15$, 17-25], and none reached genome-wide significance. A lack of replication and significance in previous research is mostly likely due to underpowered studies that have predominantly been candidate-gene focused [26, 27]. Furthermore, a comparator arm is necessary to discriminate true inter-individual variability from random and technical variability, yet very few studies included such a group, nor did they investigate or control for population stratification. This evidence to-date questions the validity of using currently available commercial genetic tests to prescribe exercise interventions.

Larger sample sizes are needed to build upon current research and to overcome random error in $\mathrm{VO}_{2}$ peak measurement at the individual level. Greater collaboration between research centres using a discovery driven approach free from pre-existing bias is warranted [26]. $\mathrm{VO}_{2}$ peak response between different population groups and training interventions along with assessing how individual factors modulate response, should also be explored [28]. The aim of this study was to use one of the largest cohorts to-date (multi-centre Predict-HIIT [7] study) to complete a GWAS to investigate genetic variants associated with $\mathrm{VO}_{2}$ peak response following exercise training interventions. In addition, we attempted to replicate candidate variants from previous studies, and aimed to build and validate a genetic prediction model for $\mathrm{VO}_{2}$ peak response (polygenic predictor score, PPS) based on the genetic data.

\section{Methods \\ Cohorts}

\section{Discovery cohort-'Predict HIIT'}

Predict-HIIT participant characteristics, recruiting and study intervention details have been previously outlined [7]. Ethical approval was obtained from the Bellberry ethical committee at the University of Queensland (\#201602-062-A-1), and from all the institutions involved. Participant data was collated from 18 exercise training interventions across eight universities from three continents. As outlined in our previous paper [7], participant change in objectively measured $\mathrm{VO}_{2}$ peak (indirect calorimetry from a graded exercise test to volitional fatigue on a treadmill or cycle ergometer) was obtained following high-volume HIIT (sessions contained $\geq 15 \mathrm{~min}$ of highintensity efforts in total, $n=225$ ), low-volume HIIT/SIT (sessions contained $<15$ min of high-intensity efforts in total, $n=76$ ), or MICT (sessions contained $30+$ minutes of continuous exercise at $64-76 \%$ maximum heart rate, $n=206$ ). The characteristics of the 507 participants from predominantly European descent used in our GWAS are outlined in Table 1 ( $24 \%$ female, age $55.9 \pm 16.9$ years, 
Table 1 Genome-wide association study participant characteristics. Mean \pm standard deviation

\begin{tabular}{|c|c|c|c|c|}
\hline & High-volume HIIT & MICT & Low-volume HIIT/SIT & All \\
\hline Participants & 225 & 206 & 76 & 507 \\
\hline Sex (male/female) & $187 / 38$ & $156 / 50$ & $42 / 34$ & $385 / 122$ \\
\hline Age (years) & $53.4 \pm 17.4$ & $61.9 \pm 12.2$ & $46.6 \pm 20.1$ & $55.9 \pm 16.9$ \\
\hline $\begin{array}{l}\text { Baseline relative } \mathrm{VO}_{2 \text { peak }} \\
(\mathrm{mL} / \mathrm{kg} / \mathrm{min})\end{array}$ & $32.1 \pm 11.5$ & $27.6 \pm 8.1$ & $30.4 \pm 13.7$ & $30.1 \pm 10.8$ \\
\hline $\begin{array}{l}\text { Relative } \mathrm{VO}_{2 \text { peak }} \text { response } \\
(\mathrm{mL} / \mathrm{kg} / \mathrm{min})\end{array}$ & $\begin{array}{l}3.4 \pm 4.1 \\
\text { Range: }-6.5 \text { to } 18.4\end{array}$ & $\begin{array}{l}2.9 \pm 3.6 \\
\text { Range: }-7.4 \text { to } 15.3\end{array}$ & $\begin{array}{l}1.9 \pm 2.8 \\
\text { Range: }-4.6 \text { to } 8.8\end{array}$ & $\begin{array}{l}* 3.0 \pm 3.8 \\
\text { Range: }-7.4 \text { to } 18.4\end{array}$ \\
\hline $\begin{array}{l}\text { Number of 'likely non } \\
\text { responders' }(>1 \text { TEM } \\
\text { below }+M C I D \text { to }<1 \text { TEM } \\
\text { below the }-M C I D)\end{array}$ & $\begin{array}{l}86(38 \%) \\
\mathrm{O}_{2 \text { peak }} \text { response }=-0.6 \pm 1.9 \\
\quad(\mathrm{~mL} / \mathrm{kg} / \mathrm{min}) \\
\text { PPS: } 2.7 \pm 2.2\end{array}$ & $\begin{array}{l}82(40 \%) \\
\mathrm{VO}_{2 \text { peak }} \text { response }= \\
-0.5 \pm 1.9(\mathrm{~mL} / \mathrm{kg} / \mathrm{min}) \\
\text { PPS: } 2.5 \pm 2.2\end{array}$ & $\begin{array}{l}42(55 \%) \\
\vee O_{2 \text { peak }} \\
\text { response }=-0.1 \pm 1.5 \\
(\mathrm{~mL} / \mathrm{kg} / \mathrm{min}) \\
\text { PPS: } 2.4 \pm 2.6\end{array}$ & $\begin{array}{l}* 210(41.4 \%) \\
\mathrm{VO}_{2 \text { peak }} \\
\text { response }=-0.43 \pm 1.9 \\
(\mathrm{~mL} / \mathrm{kg} / \mathrm{min}) \\
\mathrm{PPS}: 2.5 \pm 2.3\end{array}$ \\
\hline $\begin{array}{l}\text { Number of 'Likely } \\
\text { responders' (>1 TEM } \\
\text { above the + MCID) }\end{array}$ & $\begin{array}{l}67(30 \%) \\
\mathrm{VO}_{2 \text { peak }} \text { response }=8.4 \pm 2.8 \\
(\mathrm{~mL} / \mathrm{kg} / \mathrm{min}) \\
\text { PPS: } 6.8 \pm 3.7\end{array}$ & $\begin{array}{l}43(21 \%) \\
\mathrm{VO}_{2 \text { peak }} \text { response }=8.0 \pm 2.1 \\
(\mathrm{~mL} / \mathrm{kg} / \mathrm{min}) \\
\text { PPS: } 6.3 \pm 3.1\end{array}$ & $\begin{array}{l}12(16 \%) \\
\mathrm{VO}_{2 \text { peak }} \text { response }=6.4 \pm 0.9 \\
(\mathrm{~mL} / \mathrm{kg} / \mathrm{min}) \\
\text { PPS: } 4.8 \pm 2.3\end{array}$ & $\begin{array}{l}{ }^{* *} 122(24.1 \%) \\
\mathrm{VO}_{2 \text { peak }} \text { response }=8.1 \pm 2.4 \\
(\mathrm{~mL} / \mathrm{kg} / \mathrm{min}) \\
\text { PPS: } 6.4 \pm 2.7\end{array}$ \\
\hline $\begin{array}{l}\text { Number of 'uncertain' } \\
\text { responders }(<1 \text { TEM } \\
\text { above to }<1 \text { TEM } \\
\text { below + MCID) }\end{array}$ & $\begin{array}{l}72(32 \%) \\
\mathrm{VO}_{2 \text { peak }} \text { response }=3.6 \pm 1.0 \\
(\mathrm{~mL} / \mathrm{kg} / \mathrm{min}) \\
\text { PPS: } 3.5 \pm 2.6\end{array}$ & $\begin{array}{l}82(39 \%) \\
\mathrm{VO}_{2 \text { peak }} \text { response }=3.5 \pm 3.6 \\
\quad(\mathrm{~mL} / \mathrm{kg} / \mathrm{min}) \\
\text { PPS: } 3.4 \pm 2.9\end{array}$ & $\begin{array}{l}22(29 \%) \\
\mathrm{VO}_{2 \text { peak }} \text { response }=3.1 \pm 1.0 \\
(\mathrm{~mL} / \mathrm{kg} / \mathrm{min}) \\
\text { PPS: } 3.5 \pm 0.9\end{array}$ & $\begin{array}{l}175(34.5 \%) \\
\mathrm{VO}_{2 \text { peak }} \text { response }=3.5 \pm 0.99 \\
\quad(\mathrm{~mL} / \mathrm{kg} / \mathrm{min}) \\
\text { PPS: } 3.5 \pm 3.1\end{array}$ \\
\hline
\end{tabular}

TEMs were slightly different for each training intervention and have been outlined in Table 3

Technical error of measurement $(\mathrm{TEM})=$ multiplying mean $\mathrm{VO}_{2 \text { peak }}$ value by a previously published coefficient of variation for $\mathrm{VO}_{2 \text { peak }}$ of $5.6 \%$, Minimal Clinically Important Difference $(M C I D)=3.5 \mathrm{~mL} / \mathrm{kg} / \mathrm{min}$, Polygenic Predictor Score (PPS). *Significant difference between high-volume HIIT \& low-volume HIIT/SIT ( $P<0.05)$,

** Significant difference between high-volume HIIT, MICT \& low-volume HIIT/SIT $(P<0.05)$

$83 \%$ with pathologies and/or elderly). The deoxyribonucleic acid (DNA) extraction, preparation and genotyping are outlined below. These details varied based on the study site where the sample was collected, and whether DNA extraction and/or genotyping had already been completed prior to this study. Our quality control measures have limited bias associated with different DNA preparation, extraction methods and genotyping.

\section{Validation cohort-'Improve-HIIT'}

For replication of our results, we utilised the unpublished findings from an independent study recently performed in our laboratory (Improve-HIIT). The 'Improve-HIIT' study examined the response to highvolume HIIT by randomly allocating 40 sedentary $(<1 \mathrm{~h}$ of structured exercise each week) but apparently healthy Caucasian adults (age 18-50) to one of two groups: (i) 6 weeks of supervised high-volume HIIT (5 min warm up, 4 min $90-95 \%$ heart rate maximum followed by 3 min recovery repeated 4 times, $3 \times 1$ week) + prebiotic fibre (oligofructose-enriched inulin) supplementation ( $12 \mathrm{~g} /$ day) or (ii) 6 weeks of supervised high-volume HIIT $(3 \times /$ week $)+$ placebo (maltodextrin) supplementation $(12 \mathrm{~g} /$ day). There was no difference in the average $\mathrm{VO}_{2}$ peak response, or the inter-individual variability in $\mathrm{VO}_{2}$ peak response between study groups; as such, this study was deemed appropriate for validating findings from the Predict-HIIT GWAS. Ethical approval was obtained from the Institutional Human Research Ethics Approval committee at the University of Queensland (\#2018000398).

Each participant completed a series of tests and several measures were collated before and after the intervention. Tests relevant to this analysis included the completion of an incremental $\mathrm{VO}_{2}$ peak test to exhaustion on a treadmill (Ramped Bruce Protocol) using indirect calorimetry (Parvo Medica True One 2400 System, Parvo Medics, Inc., Sandy, UT, USA) before and after the intervention period, and provision of a saliva sample for genetic analysis (Oragene DNA collection kit, DNA Genotek, Ontario, Canada).

Genotyping, imputation and quality control were completed with the same protocol as for the PredictHIIT cohort. One sample was removed due to high missing genotyping rate, leaving 39 samples for further analysis. $\mathrm{VO}_{2}$ peak response (post intervention $\mathrm{VO}_{2}$ peak-pre intervention $\mathrm{VO}_{2}$ peak) was calculated for each participant. Fibre/placebo supplement, age, sex, body fat percentage and baseline $\mathrm{VO}_{2}$ peak were not correlated with response and were not included as covariates for analysis. Using PLINK, the top ranked loci $\left(P<1 \times 10^{-5}\right)$ from the Predict-HIIT study were compared in the Improve-HIIT study. Lower ranking 
Table 2 Validation study (Improve-HIIT) participant characteristics. Mean \pm standard deviation

\begin{tabular}{|c|c|c|c|c|}
\hline Intervention & Age (years) & $\operatorname{Sex}(M=$ male, $F=$ female $)$ & $\begin{array}{l}\text { Baseline } \mathrm{VO}_{2 \text { peak }} \\
(\mathrm{mL} / \mathrm{kg} / \mathrm{min})\end{array}$ & $\begin{array}{l}\mathrm{VO}_{\text {2peak }} \\
\text { response }(\mathrm{mL} / \\
\mathrm{kg} / \mathrm{min})\end{array}$ \\
\hline 8 weeks of maltodextrin +6 weeks of high-volume HIIT & $30.4 \pm 9.8$ & $4 \mathrm{M}, 16 \mathrm{~F}$ (20 Total) & $29.3 \pm 7.4$ & $3.7 \pm 4.7$ \\
\hline $\begin{array}{l}8 \text { weeks of oligo-fructose enriched inulin }+6 \text { weeks of } \\
\text { high-volume HIIT }\end{array}$ & $32.8 \pm 9.8$ & 5 M, 14 F (19 Total) & $35.6 \pm 5.3$ & $3.9 \pm 5.3$ \\
\hline Total & $31.6 \pm 9.8$ & 9 M, 30 F (39 Total) & $32.4 \pm 7.1$ & $3.8 \pm 5.0$ \\
\hline
\end{tabular}

loci $\left(P<1 \times 10^{-4}\right)$ were also examined between cohorts (see Table 2 for study characteristics).

\section{DNA preparation}

\section{DNA extraction from whole blood}

Genomic DNA from 58 whole blood samples [29] was extracted using a QIAamp DNA blood midi kit (Qiagen, Hilden, Germany) according to the manufacturer's instructions. The DNA samples were quantified using a Qubit fluorometer 3.0 and all samples were diluted to $100 \mathrm{ng} / \mu \mathrm{L}$ for genotyping.

\section{DNA extraction from buffy coat}

DNA from 93 buffy coats [30] was extracted using a QIAsymphony DSP DNA Mini Kit according to manufacturer's instructions [31]. The purified genomic DNA was stored at -20 degrees until genotyped.

\section{DNA extraction from saliva samples}

DNA from 289 saliva samples from two studies [32, 33] were extracted using a QIAsymphony (Qiagen) DNA MIDI Kit according to manufacturer's instructions. The yield and purity were measured using a Trinean DropSense-96. DNA from a further 93 saliva samples from 10 studies [34-43] were extracted using the protocol outlined on the DNA Genotek website [44].

\section{Genotyping}

DNA from 417 samples [29, 30, 32, 34-43] were genotyped using Illumina CoreExome chips 24v1.1 following standard protocols at the Australian Translational Genomics Centre, Princess Alexandra Hospital, Brisbane. A further 116 samples from Norway [33] were genotyped using Illumina CoreExome chips 24v1.2 at the Genomics Core Facility, NTNU. Bead intensity data was processed and normalised for each sample, and genotypes were identified using the Illumina Genome Studio software with corresponding manifest files. SNP coordinates were annotated to the GRCh37 genome build.

\section{Data quality control}

Genotypes at individual SNPs from all cohorts were merged according to the manifest and plink files. Quality control was completed separately on individual cohorts, and included assessment of missingness by individual (threshold $<5 \%$ ), missingness by genotype (threshold $<5 \%)$, Hardy-Weinberg equilibrium in controls $\left(P<1 \times 10^{-6}\right)$, extreme heterozygosity (threshold $>3$ standard deviations from mean) and identity by descent threshold at 0.2 of PI_HAT score $(\mathrm{n}=13$ excluded individuals). For each pair of related samples (PI HAT > threshold), the sample with the higher missingness rate was removed ( $\mathrm{n}=3$ excluded individuals). Along with quantitative GWAS analysis, we further defined groups based on their relative change in $\mathrm{VO}_{2}$ peak $(\mathrm{mL} /$ $\mathrm{kg} / \mathrm{min}$ ) for additional comparisons. Samples were classified as a 'likely-responder,' 'likely non-responder/adverse responder' and 'uncertain responder' based on their relative change in $\mathrm{VO}_{2}$ peak $(\mathrm{mL} / \mathrm{kg} / \mathrm{min})$ following training. As outlined in Williams et al. [7], a likely responder achieved a $\mathrm{VO}_{2 \text { peak }}$ response above one minimal clinically important difference $(3.5 \mathrm{~mL} / \mathrm{kg} / \mathrm{min})$ associated with a $10-25 \%$ improvement in survival over a 10 -year period, plus one technical error of measurement (average baseline $\mathrm{VO}_{2}$ peak multiplied by coefficient variation of 5.6\%; calculated for each study). This high threshold for response was used to increase the confidence in the 'likely responder'/likely non-responder' classification. The thresholds are provided in Table 3.

SNPs with Minor Allele Frequency (MAF) $>0.05$ were then used to perform principal component analysis (PCA) for ethnicity identification using SHELLFISH [45]. Ethnic and ancestry outliers (more than 6 standard deviations from the mean on either of the two first principal components $(\mathrm{PCs}))$ were excluded $(\mathrm{n}=10)$. Then, data was imputed with the Haplotype Reference Consortium (HRC) reference panel 1.1 [45] using the Sanger imputation server. SNPs with low imputation quality (INFO score $\leq 0.6$ ) were excluded from further analysis. In total, 26 samples were removed due to large ethnicity deviations from the group, leaving 507 samples for association testing (Table 1). Genomic inflation factor $\lambda$ and quantile-quantile $(\mathrm{Q}-\mathrm{Q})$ plots were used to compare the genome-wide distribution of the test statistic with the expected null distribution. The genomic inflation factor $\lambda$ is defined as the median of the observed chi-squared 
Table 3 Thresholds for response

\begin{tabular}{llcrc}
\hline Category & Criteria & $\begin{array}{l}\text { High-volume HIIT } \\
\text { (mL/kg/min) }\end{array}$ & MICT (mL/kg/min) & $\begin{array}{l}\text { Low-volume } \\
\text { HIIT/SIT (mL/kg/ } \\
\text { min) }\end{array}$ \\
\hline Likely responder & $>$ 1 TEM above the + MCID & $>5.3$ & $>5.0$ & $>5.2$ \\
Likely non-responder & $>$ 1 TEM below + MCID to < 1 TEM below the -MCID & -5.3 to 1.7 & -5.0 to 2.0 & -5.2 to 1.8 \\
Uncertain responders & $<$ 1 TEM above to $<$ 1 TEM below + MCID & 1.7 to 5.3 & 2.0 to 5.0 & 1.8 to 5.2 \\
\hline
\end{tabular}

Technical error of measurement $(\mathrm{TEM})=$ multiplying mean $\mathrm{VO}_{2 \text { peak }}$ value by a previously published coefficient of variation for $\mathrm{VO} \mathrm{2peak}$ of $5.6 \%$,

Minimal Clinically Important Difference (MCID) $=3.5 \mathrm{~mL} / \mathrm{kg} / \mathrm{min}$

test statistic divided by the expected median of the corresponding chi-squared distribution. A $\lambda$ close to 1 reflects no evidence of inflation, while values up to 1.10 are generally considered acceptable for a GWAS. Baseline $\mathrm{VO}_{2}$ peak, the individual study and PC6 (the 6th principal components from the PCA analysis, which was significantly associated with the phenotype) were included as covariates.

\section{Statistical analysis \\ $\mathrm{VO}_{2}$ peak response}

Normality for $\mathrm{VO}_{2}$ peak was assessed using the ShapiroWilk test. An analysis of variance was used to compare average group $\mathrm{VO}_{2}$ peak response between training interventions (high-volume HIIT, MICT, low-volume HIIT/ SIT). Variability in response was measured by the range of responses for each intervention. A chi-squared test was used to compare the proportion of likely responders, likely non-responders and those participants classified as uncertain between training groups.

\section{Association testing of independent $\mathrm{VO}_{2 \text { peak }}$ responses}

Similar to previous studies in this area [15] investigating polygenic phenotypes (i.e. $\mathrm{VO}_{2}$ peak trainability), we used a quantitative approach rather than a case-control analysis to identify variants associated with $\mathrm{VO}_{2}$ peak response. Association testing was conducted in PLINK [46], using a linear regression. Baseline $\mathrm{VO}_{2}$ peak, the individual study and the PC6 from the principal component analysis were found to be significantly associated with $\mathrm{VO}_{2}$ peak response and were included as covariates in analysis. Age and sex were not associated with the trait. Our findings did not change when age and sex were also included in the association analysis. Thus, we included covariates based on a posteriori instead of a priori knowledge. Association analyses of imputed SNPs was assessed with PLINK best-guess genotypes. Genome-wide significance was set at the standard GWAS threshold of $P<5 \times 10^{-8}$ and suggestive significance was set at $P<1 \times 10^{-5}$. The single most significant SNP (the lead SNP) was used to represent each of the loci. The cluster plots of the genotyped lead SNP, or supported genotyped SNPs of imputed lead SNP, were checked manually to eliminate poor signals. An analysis of covariance was used to compare the average $\mathrm{VO}_{2}$ peak response for each genotype of the top-ranked SNPs, including baseline $\mathrm{VO}_{2}$ peak, the individual study and PC6 as covariates. Statistical analysis was completed using SPSS (version 23.0, SPSS Inc., Chicago, IL, USA).

\section{Polygenic predictor score}

A polygenic predictor score (PPS) was calculated for each participant using the beta coefficient of the selected SNPs. The PPS was an extension of the 'summary predictor score' outlined by Bouchard et al. [15] using data from the HERITAGE study. In our study, we sought to improve on this model to ensure the 'high response training alleles'/'effect' alleles were weighted by the effect size (beta coefficient) derived from our GWAS (see Eq. 1).

$$
P P S=\sum_{i}^{k} \beta_{i} * n_{i}
$$

where $i$ is the index of the SNP in $k$ selected SNPs used to calculate the PPS. $\beta_{i}$ is the effect size (beta coefficient of linear regression) of SNP $i$ in the PPS model, $n_{i}$ is the number of effect alleles of SNP $i$.

Scores were then added across $k$ SNPs to yield a final PPS and a comparison was made between likely responders, likely non-responders and those deemed uncertain (as described earlier). To avoid over-training (inability of model to be generalised to new data), we did a tenfold cross-validation (using MultiBLUP [47]) with the discovery cohort samples and merged the results of 10 test folds for the analysis. The tenfold cross validation was to test the PPS model's ability to predict $\mathrm{VO}_{2}$ peak response in new data not related to the development of the PPS model internally.

\section{Replication of candidate loci}

The 97 loci identified as candidate loci for $\mathrm{VO}_{2}$ peak response in our recent systematic review [16] were analysed and compared with the top-ranking loci $\left(\alpha<1 \times 10^{-5}\right)$ from the Predict-HIIT study. Lead SNPs 
from all associated loci were used to calculate the PPS, as well as the 97 genetic variants found previously, were mapped to the nearest gene and submitted as a batch query to the ToppGene pathway analysis software [48]. Biological processes and pathways that appeared in both groups were selected. Genetic variants were also submitted to the GTEx Portal to identify if any SNPs were expressive quantitative trait loci (eQTL) [49].

\section{Power calculation}

Power calculations were performed using the Genomewide Complex Trait Analysis-Genomic-RelatednessBased Restricted Maximum Likelihood (GCTA-GREML) calculator [50].

\section{Results}

$\mathrm{VO}_{2}$ peak response was normally distributed. Participants included in the GWAS from high-volume HIIT interventions had a greater $\mathrm{VO}_{2}$ peak response at the group level than participants from a low-volume HIIT/SIT intervention $(1.6 \mathrm{~mL} / \mathrm{kg} / \mathrm{min}, 95 \%$ CI 0.6 to $2.5, P=0.002)$, but a comparable group $\mathrm{VO}_{2}$ peak response to participants from MICT interventions $(0.6 \mathrm{~mL} / \mathrm{kg} / \mathrm{min}, 95 \% \mathrm{CI}-0.1$ to $1.3, P=0.1)$. Participants from MICT and low-volume HIIT/SIT interventions had similar group responses $(1.0 \mathrm{~mL} / \mathrm{kg} / \mathrm{min}, 95 \% \mathrm{CI}-0.1$ to $2.0, P=0.05)$. Despite these group mean changes, there was large variability in individual $\mathrm{VO}_{2}$ peak training response within each intervention (see Fig. 1 and Table 1 for ranges). Highvolume HIIT had more likely responders than MICT ( $9 \%, 0.7$ to $17.0, \mathrm{P}=0.03$ ) and low-volume HIIT/SIT (14\%, 2.7 to 23.1, $\mathrm{P}=0.02)$. However, high-volume HIIT had similar likely non-responders to MICT $(-2 \%, 95 \%$ CI -7.2 to $11.1, P=0.7)$ and less likely non-responders to low-volume HIIT/SIT ( $-17 \%, 95 \% \mathrm{CI}-4.1$ to -29.3 , $P=0.01)$. Furthermore, high-volume HIIT had similar uncertain responders to MICT $(-7 \%, 95 \% \mathrm{CI}-2.0$ to $15.9, P=0.1)$ and low-volume HIIT/SIT $(3 \%,-9.4$ to $14.0, P=0.6)$. To establish the genetic contribution towards this variance in response to each exercise training intervention, we completed a GWAS that was adjusted for significant covariates (individual study that the participant completed, baseline $\mathrm{VO}_{2}$ peak and PC6).

No SNPs reached the typical threshold for genomewide significance $\left(P<5 \times 10^{-8}\right)$. The Q-Q plot and a genomic inflation factor of 1.002 indicated there was very minor inflation in the study (i.e. population stratification or DNA sample quality), and minor overdispersions of test statistics when compared to the null distribution (Fig. 2). Twelve loci were associated with $\mathrm{VO}_{2}$ peak response at suggestive significance $\left(P<1 \times 10^{-5}\right.$, Fig. 3 and Table 4). The most significant association was found for rs6959961 near the membrane associated guanylate kinase, WW and PDZ domain containing 2 (MAGI2) gene $\left(P=2.61 \times 10^{-7}\right)$. Homozygotes for the response allele (TT, $\mathrm{n}=93)$ had a $2.4 \mathrm{~mL} / \mathrm{kg} / \mathrm{min}$ greater $\left(P=2.8 \times 10^{-7}\right)$ average $\mathrm{VO}_{2}$ peak response than those homozygote for the non-response allele $(\mathrm{CC}, \mathrm{n}=152)$ and a $1.3 \mathrm{~mL} /$ $\mathrm{kg} / \mathrm{min}$ greater $(P=0.002)$ average $\mathrm{VO}_{2}$ peak response than heterozygotes (TC, $\mathrm{n}=262$ ). The second most significant association $\left(P=2.75 \times 10^{-7}\right)$ was found for rs730747755 near the Unc-80 Homolog, NALCN Channel Complex Subunit (UNC80) gene. Homozygotes for the response allele (AA, $\mathrm{n}=66$ ) had a $2.6 \mathrm{~mL} / \mathrm{kg} / \mathrm{min}$ greater $\left(P=1.2 \times 10^{-7}\right)$ average $\mathrm{VO}_{2}$ peak response than those homozygote for the non-response allele (GG, $n=229$ ), and a $1.8 \mathrm{~mL} / \mathrm{kg} / \mathrm{min}$ greater $\left(P=2.5 \times 10^{-4}\right)$ average $\mathrm{VO}_{2}$ peak response than heterozygotes $(\mathrm{AG}, \mathrm{n}=212)$.

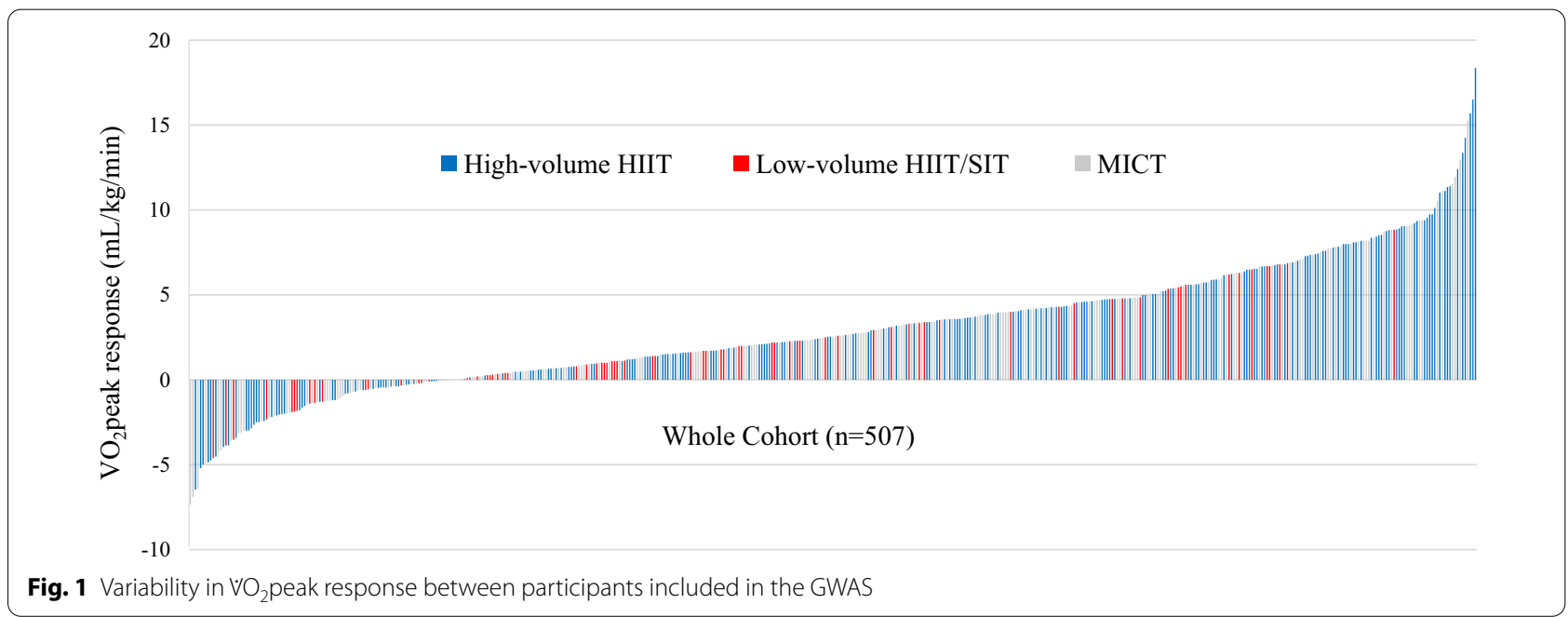




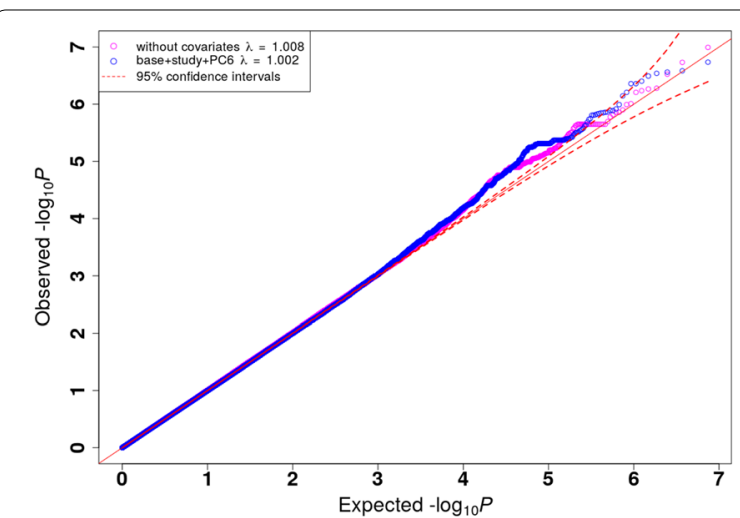

$\lambda=$ lambda, base $=$ baseline $\mathrm{VO}_{2}$ peak, study=individual study participant completed, PC6 $=6^{\text {th }}$ principal component

Fig. 2 Quantile-Quantile (QQ) plot and genomic inflation factor $\lambda$. $\lambda$ is the observed median of test statistic distribution divided by the expected median of the test statistic distribution. A genomic inflation factor greater than 1.1 indicates there may be some inflation of the GWAS P-values; resulting from factors such as population stratification or DNA sample quality. $\lambda=$ lambda, base $=$ baseline $\mathrm{VO}_{2}$ peak, study $=$ individual study participant completed, $P C 6=6$ th principal component

A tenfold cross validation found the Pearson correlation coefficient between subject polygenic predictor score (PPS) and $\mathrm{VO}_{2}$ peak response (likely responder, likely non responder or uncertain) was not significant $\left(R^{2}=0.027, P\right.$-value $=0.76$, see Fig. 4). Similarly, the PPS was not able to predict $\mathrm{VO}_{2}$ peak training response in the validation (Improve-HIIT) cohort $\left(R^{2}=0.001\right.$, $P=0.8$ ). None of the 12 lead SNPs from our GWAS had a $P$-value $<0.05$ in the Improve-HIIT study. Furthermore, from the 992 variants with a $P$-value $<1 \times 10^{-4}$ in the Predict-HIIT cohort, a correlation of beta coefficients in the discovery (Predict-HIIT) cohort and the Improve-HIIT cohort was found to be significant $\left(\mathrm{R}^{2}=0.156, P\right.$-value $\left.=7.62 \times 10^{-7}\right)$. This suggests these variants in the Improve-HIIT cohort have a significant similar trend of effect as they do in the Predict-HIIT cohort.

Whilst none of the 12 lead SNPs from our GWAS validated SNPs found in previous research, several of our lead 12 SNPs were found near genes that are in similar biological pathways and processes to predictor genes found in previous research (Table 5). Additionally, we were able to validate a number of SNPs from previous research at a nominal level ( 4 SNPs at $P$-value $<0.05$, see Table 6). Furthermore, we found several SNPs to be eQTL in tissues that may influence training adaptations. For example, rs11647343, is an eQTL of zinc finger DHHC-type palmitoyltransferase 7(ZDHHC7) in whole blood $\left(P=1.8 \times 10^{-5}\right)$. The SNP, rs2657147, is

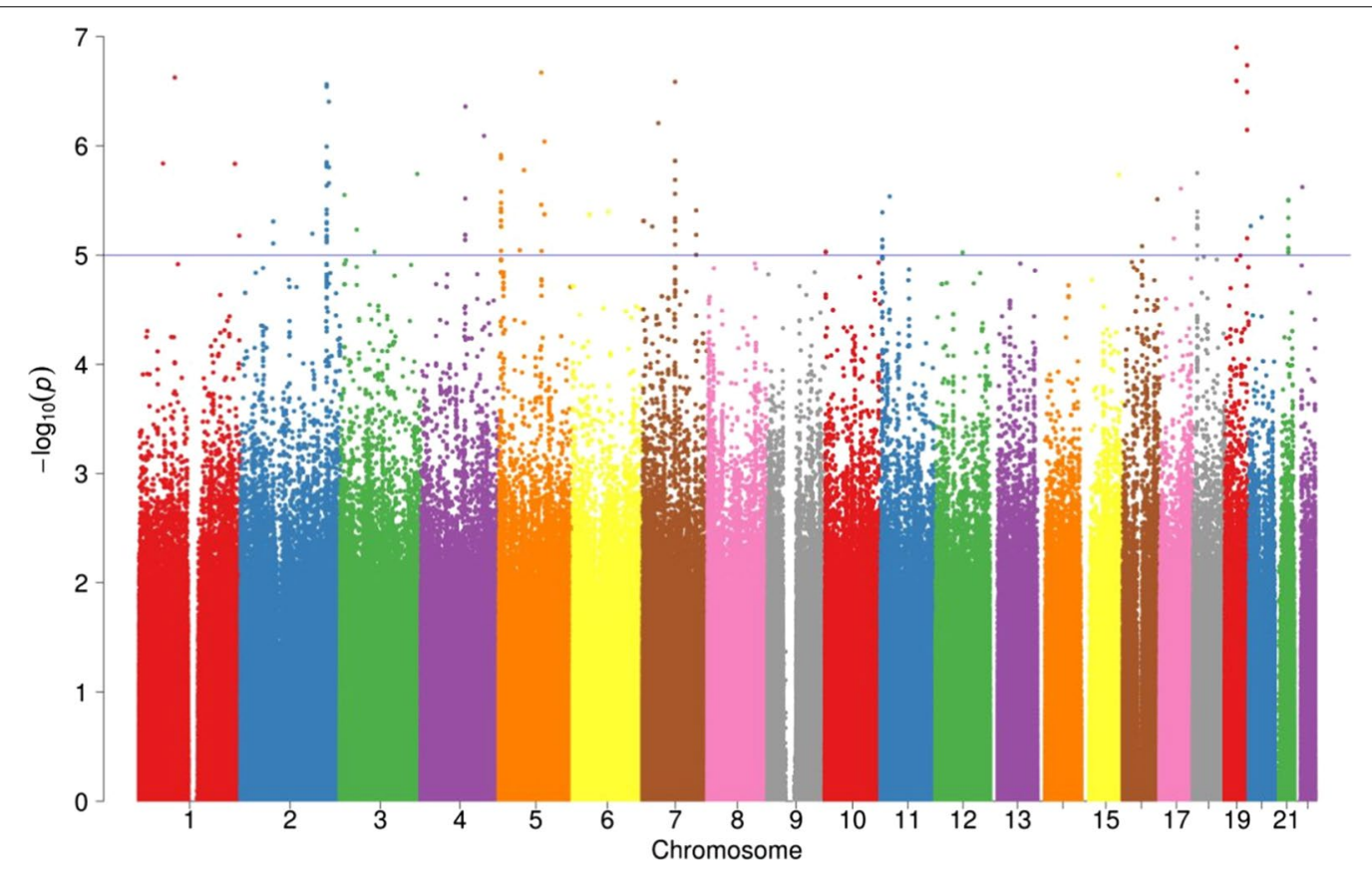

Fig. 3 Manhattan Plot of whole Training Cohort. The X-axis represents genomic coordinates, with the negative logarithm of the association p-value for each variant displayed on the $Y$-axis. Different chromosomes are shown with different colours. The blue line indicates the suggestive significance threshold $1 \times 10^{-5}$ 
Table 4 Lead SNP at each locus showing a trend for association with $V O 2$ peak response

\begin{tabular}{|c|c|c|c|c|c|c|c|c|}
\hline SNP & CHR & BP & P-value & BETA & Closet Gene & Effect allele & Other allele & MAF \\
\hline rs73074755 & 2 & 210627971 & $2.75 \times 10^{-7}$ & 1.17 & $\begin{array}{l}\text { UNC80 } \\
\text { Unc-80 Homolog, NALCN Channel Complex Subunit }\end{array}$ & A & G & 0.34 \\
\hline rs16875411 & 5 & 5359876 & $1.22 \times 10^{-6}$ & -1.56 & $\begin{array}{l}\text { ADAMTS16 } \\
\text { A Disintegrin-Like And Metalloprotease (Reprolysin Type) } \\
\text { With Thrombospondin Type } 1 \text { Motif, } 16\end{array}$ & A & G & 0.12 \\
\hline rs2236368 & 6 & 41309353 & $4.27 \times 10^{-6}$ & 1.97 & $\begin{array}{l}\text { TFEB } \\
\text { Transcription Factor EB }\end{array}$ & A & G & 0.068 \\
\hline rs111346648 & 7 & 2322104 & $4.86 \times 10^{-6}$ & 3.27 & $\begin{array}{l}\text { AMZ1 } \\
\text { Archaelysin Family Metallopeptidase } 1\end{array}$ & G & A & 0.026 \\
\hline rs6959961 & 7 & 79297997 & $2.61 \times 10^{-7}$ & 1.19 & $\begin{array}{l}\text { MAGI2 } \\
\text { Membrane Associated Guanylate Kinase, WW And PDZ } \\
\text { Domain Containing } 2\end{array}$ & $\mathrm{~T}$ & C & 0.44 \\
\hline rs 2657147 & 11 & 4573236 & $4.08 \times 10^{-6}$ & -1.08 & $\begin{array}{l}\text { OR52M1 } \\
\text { Olfactory Receptor Family } 52 \text { Subfamily M Member } 1\end{array}$ & G & A & 0.37 \\
\hline rs145056992 & 11 & 23069615 & $2.92 \times 10^{-6}$ & 2.89 & $\begin{array}{l}\text { CCDC179 } \\
\text { Coiled-Coil Domain Containing } 179\end{array}$ & G & A & 0.036 \\
\hline rs79687662 & 15 & 92921291 & $1.85 \times 10^{-6}$ & 2.33 & $\begin{array}{l}\text { IQGAP1 } \\
\text { IQ MotifContaining GTPase Activating Protein } 1\end{array}$ & C & $\mathrm{T}$ & 0.052 \\
\hline rs11647343 & 16 & 84454267 & $3.10 \times 10^{-6}$ & 1.12 & $\begin{array}{l}\text { ATP2C2 } \\
\text { ATPase Secretory Pathway Ca2 + Transporting } 2\end{array}$ & C & A & 0.32 \\
\hline rs11874598 & 18 & 11064859 & $4.02 \times 10^{-6}$ & 1.02 & $\begin{array}{l}\mathrm{PIEZO} 2 \\
\text { Piezo Type Mechanosensitive lon Channel Component } 2\end{array}$ & C & $\mathrm{T}$ & 0.47 \\
\hline rs149323705 & 20 & 30964328 & $4.52 \times 10^{-6}$ & 4.42 & $\begin{array}{l}\text { ASXL1 } \\
\text { Additional Sex Combs Like 1, Transcriptional Regulator }\end{array}$ & $\mathrm{T}$ & $C$ & 0.014 \\
\hline rs73193458 & 21 & 33375476 & $3.13 \times 10^{-6}$ & 1.49 & $\begin{array}{l}\text { CLDN14 } \\
\text { Claudin } 14\end{array}$ & A & G & 0.14 \\
\hline
\end{tabular}

Genome build GRCH37, chromosome (CHR), single nucleotide polymorphism (SNP), physical position (BP), odds ratio (OR), responder/non-responder allele (A1), minor allele frequency (MAF)

an eQTL of tripartite motif containing 68 (TRIM68) in subcutaneous adipose tissue $\left(P=4.8 \times 10^{-8}\right)$.

The GCTA power calculator found a cohort of 2960 samples would have $80 \%$ power to detect a quantitative trait with a true heritability of $30 \%$.

\section{Discussion}

To our knowledge this is one of the largest multi-centre GWAS to investigate CRF response following exercise training. Compared to previous genetic studies in this field of research, we were able to user newer technology and methodologies that increased the validity and accuracy of our results. Across the 507 participants and irrespective of the intervention completed, there was large variability in individual $\mathrm{VO}_{2}$ peak response to highvolume HIIT, MICT and low-volume HIIT/SIT. We were unable to identify genetic variants at a genome-wide significant level that explained this variability in response to each training intervention. However, 12 SNPs were found at a suggestive level of significance and warranted further investigation. Several of our lead SNPs seemed possible candidate genes for predicting $\mathrm{VO}_{2}$ peak response due to their association with previously identified predictor genes, and related biological pathways and processes that may influence training adaptations.

The most significantly associated SNP, MAGI2, can influence neuronal cell activin-mediated signalling, and may supress AKT Serine/Threoine Kinase 1 (AKT1) activation [51]. $A K T 1$ is a $\mathrm{VO}_{2}$ peak response predictor gene identified from previous research, and is one of three genes from the protein kinase B family that can influence growth, differentiation and metabolism [52]. The SNP, rs1130214, found near AKT1, was significantly associated $(\mathrm{P}<0.05)$ with $\mathrm{VO}_{2}$ peak response in previous research [52] and was found at a nominal level in our Predict-HIIT cohort $(P=0.06)$. One of our lead SNPs, rs79687662, is found near the IQ Motif Containing GTPase Activating Protein 1 (IQGAP1) gene. IQGAP1 and $A K T 1$ genes are both found in the E-cadherin signalling in the nascent adherens junction biological pathway, and together with Transcription Factor Hypoxia-Inducible Factor-1 (HIF1A) and Neuropilin 2 (NRP2) (predictor genes from previous research), are found in the signalling events mediated by the Vascular Endothelial Growth Factor Receptor 1 (VEGFR1) and Vascular Endothelial Growth Factor Receptor 2 (VEGFR2) biological pathway. Furthermore, a rat model 


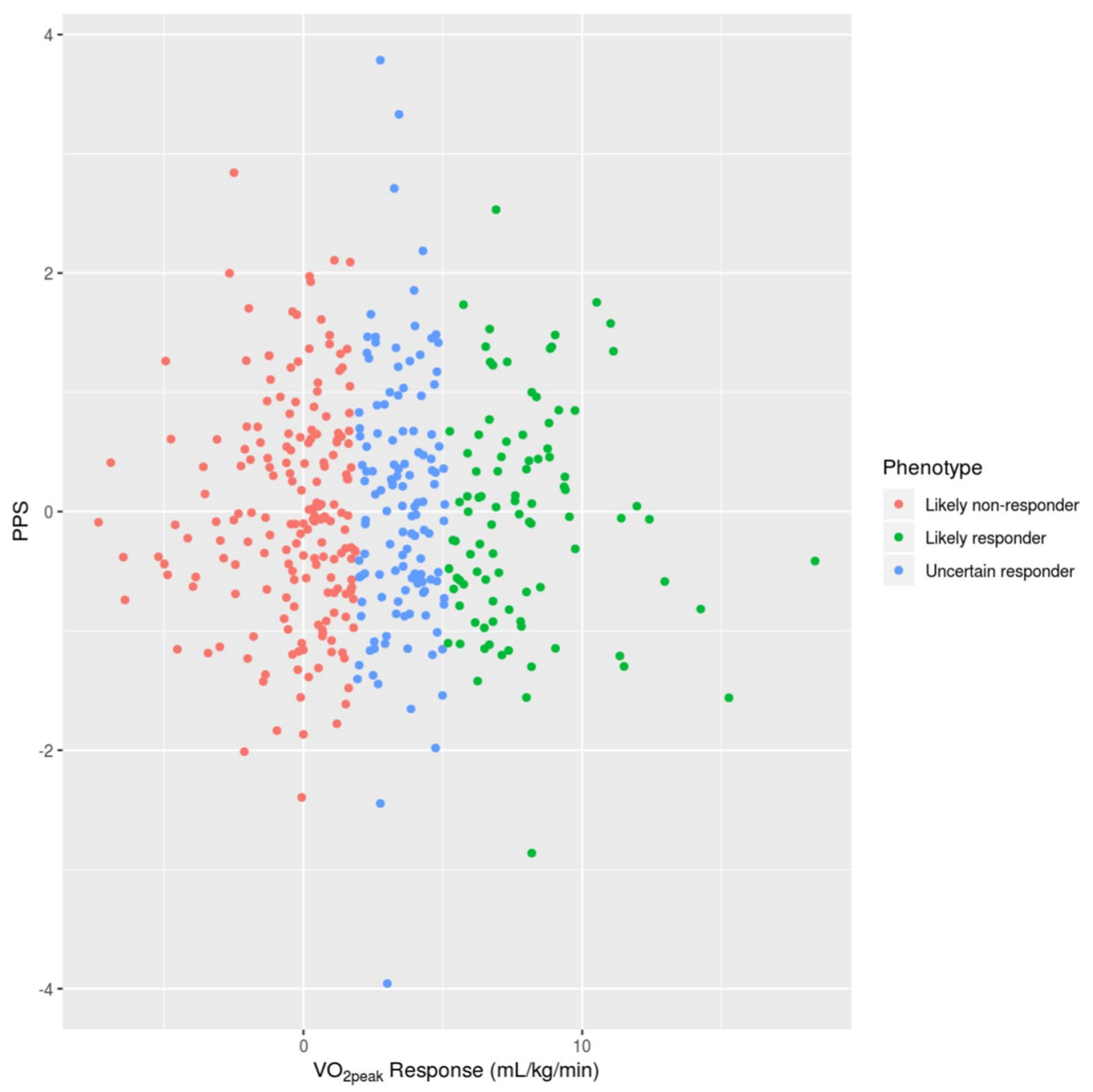

Fig. 4 Tenfold cross validation - no correlation between Polygenic Predictor Score (PPS) and $\mathrm{VO}_{2}$ peak response $\left(R^{2}=0.027, P=0.76\right)$. Red, green and blue dots represent likely non-responders, likely responders and uncertain responders, respectively

found the catenin (cadherin-associated protein) gene was upregulated in higher responders to HIIT, which helps to regulate angiogenesis, neurogenesis and tissue development [53]. Another recent rodent study found loss of Iqgap1 may lead to defective $A K T$ and Extracellular Signal-Regulated Kinase 1/2 (ERK1/2) signalling and impaired cardiomyocyte hypertrophy [54].

Our second strongest associated lead SNP, rs730747755, is found near the Unc-80 Homolog, NALCN Channel Complex Subunit (UNC80) gene. UNC80 is a gene that contributes to a large ion channel complex (the 'NALCN channelosome"), which includes the Sodium Leak Channel, Non Selective (NALCN) gene [55]. NALCN is a $\mathrm{VO}_{2}$ peak response predictor gene found in previous research [18], and similar to the $U N C 80$ gene, may influence the resting membrane potential of neuronal cells
[55]. There is evidence that genes encoding the NALCN channelosome may contribute to the susceptibility for several diseases, including cardiac diseases, some cancers and psychiatric disorders [56].

Two of our associated lead SNPs were found near genes related to peroxisome proliferator-activated (PPAR) activity. The SNP rs14932370 is found near the ASXL Transcriptional Regulator 1 (ASXL1) gene. Overexpression of $A S X L 1$ may reduce adipogenesis by decreasing Peroxisome Proliferator-Activated Receptor $y$ (PPARy) activity [57]. The SNP, rs2236368, is found near the Transcription Factor EB (TFEB) gene. TFEB may regulate mitophagy, and in addition to Peroxisome Proliferator-Activated Receptor Gamma, Coactivator 1 alpha (PGC-1 $\alpha)$, is considered important for mitochondrial biogenic regulation [58]. TFEB may also regulate 
Table 5 Gene interactions and common biological pathways and processes between this study and previous findings

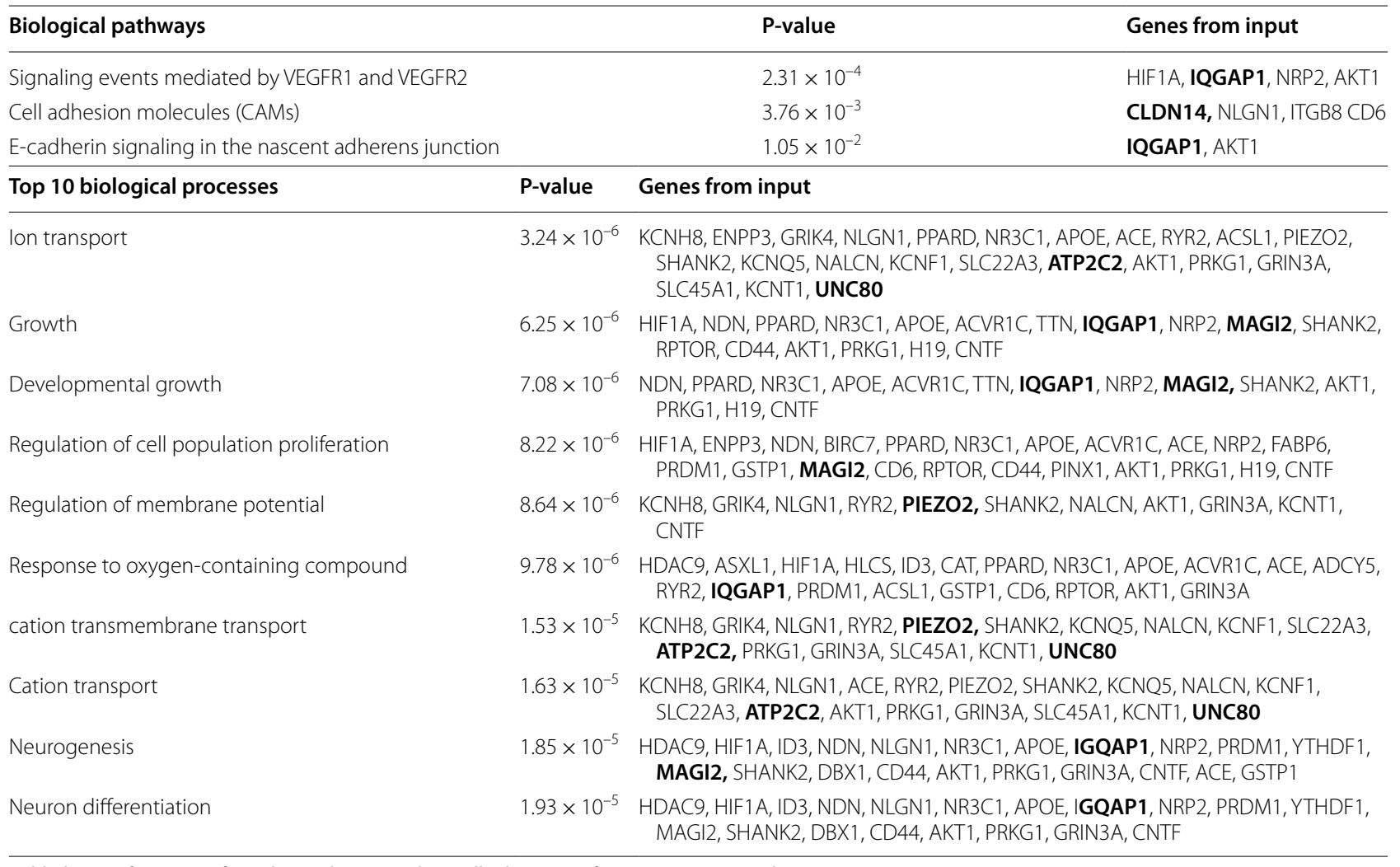

Bolded genes from input from the Predict-HIIT cohort. All other genes from previous research

Table 6 Findings from previous studies found significant at a nominal level in the Predict HIIT study

\begin{tabular}{|c|c|c|c|c|c|c|c|}
\hline SNP & Closest gene & CHR & Beta coefficient & $\begin{array}{l}P \text {-value in } \\
\text { Predict -HIIT } \\
\text { cohort }\end{array}$ & Author & $\begin{array}{l}\text { Responder allele/ } \\
\text { non-responder } \\
\text { allele in previous } \\
\text { research }\end{array}$ & $\begin{array}{l}\text { Responder allele/non- } \\
\text { responder allele in } \\
\text { Predict-HIIT study }\end{array}$ \\
\hline rs10751308 & $\begin{array}{l}\text { SHANK2 } \\
\text { SH3 and multiple } \\
\text { ankyrin repeat } \\
\text { domains } 2\end{array}$ & 11 & 0.62 & 0.02 & Gosh et al. [18] & Unknown & T allele (+) \\
\hline rs10921078 & $\begin{array}{l}\text { RGS18 } \\
\text { regulator of G protein } \\
\text { signaling } 18\end{array}$ & 1 & 0.68 & 0.02 & Bouchard et al. [15] & G allele (-) & $\mathrm{G}(-)$ \\
\hline rs1535628 & $\begin{array}{l}\text { GRIN3A } \\
\text { glutamate ionotropic } \\
\text { receptor NMDA type } \\
\text { subunit } 3 A\end{array}$ & 9 & 1.01 & 0.02 & Bouchard et al. [15] & Unknown & $A(+)$ \\
\hline rs2003298 & $\begin{array}{l}\text { NLGN1 } \\
\text { neuroligin } 1\end{array}$ & 2 & 0.47 & 0.04 & Bouchard et al. [15] & A allele (+) & $\mathrm{T}(+)$ \\
\hline
\end{tabular}

CHR, Chromosome; SNP, single nucleotide polymorphism

insulin sensitivity, glucose homeostasis and lipid oxidation [59]. Overexpression of TFEB may increase mitochondrial biogenesis and ATP production in skeletal muscle, independently from PCG-1 $\alpha$ [59]. A study also found PGC- $1 \alpha$ expression can be increased through the dephosphorylation and nuclear translocation of TFEB [60]. With these points in mind, a recent study found completing two high-intensity exercise sessions within a short time frame $(2 \mathrm{~h})$ increased the nuclear abundance of TFEB and the transcription of PCG- $1 \alpha$ 
in 8 healthy young men [61]. Furthermore, overexpression of PGC- $1 \alpha$ has been associated with improved $\mathrm{VO}_{2}$ peak at baseline and following endurance training in several studies $[62,63]$.

Several of our other associated SNPs are found in the same biological processes and pathways to variants identified in previous research [16]. The SNP rs73193458 is found near the Claudin 14 (CLDN14) gene, and together with previously identified $\mathrm{VO}_{2 \text { peak }}$ response predictor genes (Neuroligin 1 (NLGN1), Integrin Subunit Beta 8 (ITGB8) and Cluster of Differentiation 6 (CD6)) is involved in the cell adhesion molecules (CAMs) biological pathway. The SNP rs11874598 found near the Piezo Type Mechanosensitive Ion Channel Component 2 (PEIZO2) gene (which is a mechanosensitive ion channel involved in touch, proprioception, and respiratory function [64]); and rs11647343 found near the ATPase Secretory Pathway Ca2 + Transporting 2 (ATP2C2) gene (which is related to nucleotide binding and calcium transporting ATPase activity and cardiac conduction [64]); along with several other genes identified from previous studies, are involved in cation transmembrane transport biological processes. We also found rs11647343 and rs2657147 to be eQTLs of genes associated with whole blood (ZDHHC7) and subcutaneous tissue (TRIM68), respectively [49]. In mice, $Z D H H C 7$ plays a role in glucose transporter type 4 (Glut4) palmitoylation, contributing to glucose homeostasis [65], possibly contributing to metabolic adaptions required for $\mathrm{VO}_{2}$ peak improvements. TRIM68 variants have been associated with early onset obesity [66] and is upregulated following aerobic exercise [67]. TRIM68 is associated with ubiquitination [67] and may potentially play a role in proteolytic activity and exercise induced muscle damage. Body composition has been associated with exercise capacity, including maximal workload and oxygen uptake $[68,69]$. However, more work is needed to go beyond association and to identify causal variants/genes. Future functional studies are needed.

\section{Validation}

We created a PPS from the top-12 associated loci from the discovery cohort to identify who was more likely to be a responder or non-responder to different forms of training. It was hypothesised that those identified as a lower responder may need a greater training dose than reported in our study to elicit a clinically meaningful response, or other environmental influences may need to be considered. For example, Montero and Lundby [4] have shown non-responders can become responders by increasing the dose of exercise training. Despite many of the suggestively associated SNPs showing a strong connection to previously identified genes, processes and pathways that may influence training adaptions to exercise, there was no significant correlation between the PPS score and $\mathrm{VO}_{2}$ peak response following the tenfold crossvalidation. The variants and model could not accurately explain the variance in $\mathrm{VO}_{2}$ peak response or predict who may be a lower or higher responder to each of the training interventions. Likewise, an independent cohort validation, from the Improve-HIIT study, did not support an association of the lead 12 SNPs with variance in $\mathrm{VO}_{2}$ peak response when considered individually, or in the PPS model. This may be due to a relatively small sample size, or in fact that genetics plays a smaller role than previous research has alluded to. Our power calculation found we need at least 2960 samples to detect signals of common variants with a heritability of $30 \%$.

Additionally, we were unable to replicate variants ( $\mathrm{VO}_{2}$ peak response predictor genes) identified from previous research [16] at a genome wide or suggestive level of significance. However, we were able to replicate several genetic variants from previous research at a nominal significance level within the Predict-HIT cohort, including: rs10751308, rs10921078, rs1535628 and rs2003298 $(\mathrm{P}<0.05)$. Two of these SNPs (rs10921078 and rs2003298) had the same 'response' allele in the PredictHIIT cohort and previous research [15]. These SNPs warrant investigation in future studies. Furthermore, a significant Pearson correlation coefficient was found for the beta coefficient of variants with a $P$-value $<1 \times 10^{-4}$ in the Predict-HIIT cohort the Improve-HIIT cohort. This indicates general effects of the loci (as a group) exist, and a larger sample size may detect many of these effects as statistically significant.

\section{Limitations}

Several limitations may have prevented the finding of more significant associations and validating the proposed PPS model. Firstly, $\mathrm{VO}_{2}$ peak response is considered a complex trait that may result from multiple interactions between genes (epistasis) and epigenetic changes that can affect gene expression [27]. This was made evident with several of the lead Predict-HIIT SNPs sharing common biological pathways and processes to predictor genes identified from previous studies. Larger sample sizes than reported in our study (tens of thousands) are often needed to investigate these gene interactions via a GWAS, and to identify rare variants that may be contributing to overall response [70, 71]. A lack of detail in previous publications prevented some select variants from being replicated. Previous studies have predominantly been candidate-gene focused, and similar to our study, have lacked the necessary statistical power [16]. The validation study also lacked statistical power and the population studied was different to the Predict-HIIT study. 
The Predict-HIIT study included a mix of healthy, young, older and clinical European population groups from studies with a variety of exercise doses; whereas the validation study was a high volume HIIT intervention on young, healthy but inactive predominantly Caucasian adults, and included a nutrition intervention. Previous studies have predominantly investigated endurance interventions, including participants from a mix of nationalities, and mainly inactive but healthy populations [16]. Moreover, there may be differences in the accuracy of findings between studies based on participant compliance to study protocols. These factors may have influenced the gene expression and the significance of variants discovered in previous research, the validation study and the Predict-HIIT study. If our study had a larger sample size, we could have stratified our analysis to see if associations were different when clustered according to healthy and clinical populations, and training doses. We tried to combat this by including significant covariates in our GWAS model, including the individual study.

Despite limited research, the declining cost of genetic testing has created an abundance of direct-to-consumer (DTC) DNA testing companies [71]. These testing companies often base recommendations on single or very few genes. For example, Alpha-actinin-3 (ACTN3) is a common 'fitness gene' found in many DTC tests, whereby consumers are encouraged to modify the intensity, volume or frequency of their exercise training to suit their ACTN3 genotype. Potential ACTN3 'genotype-based training protocols' for strength and endurance training improvements have been outlined in previous research [72]. For $\mathrm{VO}_{2}$ peak improvement, the authors suggest RR allele homozygotes and RX allele heterozygotes are resistant to muscle damage and better suited to HIIT; whereas $\mathrm{XX}$ allele homozygotes have lower skeletal muscle function and poorer recovery, and subsequently are better suited to MICT [72]. Our analysis and other research has shown that exercise-related phenotypes, such as change in $\mathrm{VO}_{2}$ peak response, is a polygenic trait where multiple genes influence various cellular pathways $[3,14]$ and each gene may contribute only a small percentage to the overall change $[16,26,73]$. We have established that the significance of these genes and associated variants remain uncertain, questioning the importance of genetics in predicting individual response and the validity of commercial tests reliant on limited variants used for personalised exercise prescription.

An even larger study with more participants is needed to advance this field of research. In other areas of genetic research, this is achieved by combining datasets and completing a meta-analysis of many genome-wide association studies. As outlined by Zeggini and Ionnidis
[74], combining many GWAS datasets would require a consortium with various institutions and laboratories combining to develop a robust protocol that addresses selection bias, quality control, heterogeneity of populations studied and the replication of biologically plausible previous findings. Based on our findings, we have calculated that at least 2960 participants would need to be included in well-controlled exercise interventions to measure $\mathrm{VO}_{2}$ peak response. Future research should also focus on more than just the genome by using epigenomics, transcriptomics and metagenomics. Having large datasets with this information may help to identify with greater confidence the gene and pathway interactions, and epigenetic changes resulting from environmental influences [75]. Analysis of how exercise dose and quantitative traits including diet, sleep, recovery between training sessions, clinical conditions (e.g. coronary artery disease, type 2 diabetes) and how the microbiome may affect epistasis could also be explored. The Athlome Project Consortium is a collaborative initiative between several institutions to find genetic variants associated with athletic performance [76]. A similar concept could be developed specifically for finding genetic variants associated with $\mathrm{VO}_{2}$ peak response in non-athletes to aerobic training interventions.

\section{Conclusions}

In conclusion, we found 12 novel genetic variants associated with $\mathrm{VO}_{2}$ peak response in the Predict-HIIT study group. These SNPs have common biological pathways and processes to previous research findings, but could not be replicated in a small independent study. Furthermore, cross-validation found the PPS created from the top-associated SNPS did not show significant correlation with whom was likely to be a responder or nonresponder to exercise training. Heterogeneity and a lack of power in the discovery (Predict-HIIT) and validation (Improve-HIIT) cohorts may have prevented lead SNPs from being reproduced between studies. Our results highlight the possible risks associated with predictive scores for complex traits. Larger sample sizes with wellprescribed, controlled and accurately measured exercise interventions are required to identify rare variants, gene interactions and epigenetic changes that may influence gene expression and $\mathrm{VO}_{2}$ peak response, and to find the ideal exercise dose to negate non-response. Ongoing research and validation of current and previous findings is needed to confirm if genetics does play a large role in $\mathrm{VO}_{2}$ peak response variance, and whether genomic predictors for $\mathrm{VO}_{2}$ peak response trainability can inform evidence-based clinical practice. 


\begin{abstract}
Abbreviations
ACTN3: Alpha-actinin-3; AKT1: AKT Serine/Threoine Kinase 1; ASXL1: ASXL Transcriptional Regulator 1; ATP2C2: ATPase Secretory Pathway Ca2 + Transporting 2; CAMs: Cell adhesion molecules; CD6: Cluster of Differentiation 6; CLDN14: Claudin 14; DNA: Deoxyribonucleic acid; DTC: Direct to consumer; eQTL: Expression quantitative trait loci; ERK1/2: Extracellular Signal-Regulated Protein Kinase; Glut4: Glucose Transporter Type 4; GCTA-GREML: The Genomewide Complex Trait Analysis - Genomic-Relatedness-Based Restricted Maximum Likelihood; GWAS: Genome Wide Association Study; HERITAGE: HEalth, Rlsk factors, exercise Training and GEnetics; HIF1A: Hypoxia-Inducible Factor 1-Alpha; HIIT: High intensity interval training; IQGAP1: IQ Motif Containing GTPase Activating Protein 1; ITGB8: Integrin Subunit Beta 8; MICT: Moderate intensity continuous training; NALCN: Sodium Leak Channel, Non-Selective; NRP2: Neuropilin 2; PC6: Principal Component Six; PEIZO2: Piezo Type Mechanosensitive Ion Channel Component 2; PGC-1a: Peroxisome proliferatoractivated receptor gamma coactivator 1-alpha; PPAR: Peroxisome proliferatoractivated receptor; PPARy: Peroxisome proliferator-activated receptor $y$; PPS: Polygenic predictor score; QQ: Quantile-quantile; SIT: Sprint interval training; SNPs: Single nucleotide polymorphisms; TFEB: Transcription Factor EB; TRIM68: Tripartite Motif Containing 68; UNC80: Unc-80 Homolog, NALCN Channel Complex Subunit; VEGFR1: Vascular Endothelial Growth Factor Receptor-1; VEGFR2: Vascular Endothelial Growth Factor Receptor-2; VO2peak: Cardiorespiratory fitness (maximal oxygen uptake); ZDHHC7: Zinc Finger DHHC-Type Palmitoyltransferase 7.
\end{abstract}

\section{Acknowledgements}

Professor Ben Levine for providing access to participants from his study, Dr Sarah Voisin for guidance and manuscript edits, and Professor Jian Yang for his assistance with GTCA-GREML calculator.

\section{Authors' contributions}

$\mathrm{CW}, \mathrm{ZL}, \mathrm{NH}, \mathrm{RL}, \mathrm{JC}$ and NE contributed to the conception and design of the study. CW collated the data for the Predict-HIIT and Improve-HIIT study and completed baseline statistical analysis of data. CW wrote the first draft of the manuscript. ZL completed the genome wide association study for the PredictHIIT study. ZL completed the cross-validation analysis of the Predict-HIIT study, and validation analysis using data from the Improve-HIIT study. NH provided assistance with manuscript revisions, and guidance with statistical analysis. RL provided guidance with manuscript improvements and statistical analysis. IC, $N H$, JT, TG, EC, JR, RF, MJ, JL, MF, BG, JB, CH, SS, SK, SJ, EC, PB, VC, NP, EH, UW, AB, $D S, D B, I P, X Y, M B, L H, L G, K A$ were investigators involved with the Predict-HIIT study analysis. All authors read and approved the final manuscript.

\section{Funding}

This research was made possible from the funding received through the Collaborative Research Network for Advancing Exercise \& Sports Science (CRN-AESS) - Bond University, Robina, Australia. NH was supported by a PhD stipend also provided by Bond University CRN-AESS. This research was supported by infrastructure purchased with Australian Government EIF Super Science Funds as part of the Therapeutic Innovation Australia-Queensland Node project (LG).

\section{Availability of data and materials}

The datasets used and/or analysed during the current study are available from the corresponding author on reasonable request.

\section{Declarations}

\section{Ethics approval and consent to participate}

Predict-HIIT study: Ethical approval was obtained from the Bellberry ethical committee at the University of Queensland (\#2016-02-062-A-1), and from all the institutions involved. Improve HIIT study: Ethical approval was obtained from the Institutional Human Research Ethics Approval committee at the University of Queensland (\#2018000398).

\section{Consent for publication}

Not applicable.

\section{Competing interests}

The authors declare that they have no conflicts of interest that are relevant to this research paper.

\section{Author details}

${ }^{1}$ Centre for Research on Exercise, Physical Activity and Health, School of Human Movement and Nutrition Sciences, University of Queensland, St. Lucia, Brisbane, QLD, Australia. ${ }^{2}$ Translational Genomics Group, Institute of Health and Biomedical Innovation, Woolloongabba, Brisbane, QLD, Australia. ${ }^{3}$ Faculty of Health Sciences and Medicine, Bond University, Robina, QLD, Australia. ${ }^{4}$ Queensland University of Technology (QUT), Centre for Genomics and Personalised Health, Genomics Research Centre, School of Biomedical Sciences, Institute of Health and Biomedical Innovation, Kelvin Grove, Brisbane, QLD, Australia. ${ }^{5}$ School of Kinesiology and Health Studies, Queen's University, Kingston, ON, Canada. Institute for Health and Sport (iHeS), Victoria University, Melbourne, VIC, Australia. ${ }^{7}$ Cardiac Exercise Research Group (CERG), Department of Circulation and Medical Imaging, Faculty of Medicine, Norwegian University of Science and Technology, Trondheim, Norway. ${ }^{8}$ Caring Futures Institute, SHAPE Research Centre, Exercise Science and Clinical Exercise Physiology, College of Nursing and Health Sciences, Flinders University, Adelaide, SA, Australia. ${ }^{9}$ School of Health and Exercise Sciences, University of British Columbia, Kelowna, BC, Canada. ${ }^{10}$ Internal Medicine, Institute for Exercise and Environmental Medicine, University of Texas Southwestern Medical Center, Dallas, TX, USA. "'Department of Physiology, Radboud University Medical Center, Nijmegen, Netherlands. ${ }^{12}$ Department of Cardiology, Antwerp University Hospital, Antwerp, Belgium. ${ }^{13}$ Department of Rehabilitation Sciences - Research Group for Rehabilitation in Internal Disorders, Catholic University of Leuven, Leuven, Belgium. ${ }^{14}$ Baker Heart and Diabetes Institute, Melbourne, VIC, Australia. ${ }^{15}$ Australia Institute for Musculoskeletal Sciences (AIMSS), Melbourne, VIC, Australia. ${ }^{16}$ School of Medical and Health Sciences, Edith Cowan University, Joondalup, WA, Australia. ${ }^{17}$ Department of Cardiology, St. Olavs Hospital, Trondheim, Norway. ${ }^{18}$ Guy's and St Thomas' NHS FoundationTrust and King's College London, London, UK. ${ }^{19}$ Department of Sport and Health Sciences, University of Exeter, Exeter, UK. ${ }^{20}$ Department of Sport, Movement and Health, University of Basel, Basel, Switzerland.

Received: 14 January 2021 Accepted: 5 May 2021

Published online: 13 May 2021

\section{References}

1. Kodama S, Saito K, Tanaka S, Maki M, Yachi Y, Asumi M, et al. Cardiorespiratory fitness as a quantitative predictor of all-cause mortality and cardiovascular events in healthy men and women: a meta-analysis. JAMA. 2009;301(19):2024-35.

2. WHO. Chronic Diseases and Health Promotion: the World Health Organisation; 2015. http://www.who.int/chp/en/. Accessed 20 January 2019.

3. Pickering C, Kiely J. Understanding personalized training response: can genetic assessment help? Open Sports Sci J. 2017;10:191-213.

4. Montero D, Lundby C. Refuting the myth of non-response to exercise training: "non-responders" do respond to higher dose of training. JAP. 2017;595:3377-87.

5. Bonafiglia JT, Rotundo MP, Whittall JP, Scribbans TD, Graham RB, Gurd $B J$. Inter-individual variability in the adaptive responses to endurance and sprint interval training: a randomized crossover study. Plos One. 2016;11(12):e0167790.

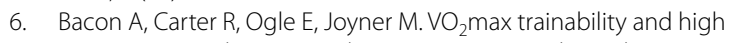
intensity interval training in humans: a meta-analysis. PloS One. 2013;8(9):e73182

7. Williams CJ, Gurd BJ, Bonafiglia JT, Voisin S, Li Z, Harvey N, et al. A multicenter comparison of VO2peak trainability between interval training and moderate intensity continuous training. Front Physiol. 2019;10:19.

8. Gist NH, Fedew MV, Dishman RK, Cureton KJ. Sprint interval training effects on aerobic capacity: a systematic review and meta-analysis. J Sports Med. 2014;44:269-79.

9. Boff W, da Silva AM, Farinha JB, Rodrigues-Krause J, Reischak-Oliveira A, Tschiedel B, et al. Superior effects of high-intensity interval vs. moderateintensity continuous training on endothelial function and cardiorespiratory fitness in patients with type 1 diabetes: a randomized controlled trial. Front Physiol. 2019;10:450. 
10. Bouaziz W, Malgoyre A, Schmitt E, Lang PO, Vogel T, Kanagaratnam L. Effect of high-intensity interval training and continuous endurance training on peak oxygen uptake among seniors aged 65 or older: a metaanalysis of randomized controlled trials. Int J Clin Pract. 2020;74:e13490.

11. Xie B, Yan X, Cai X, Li J. Effects of high-intensity interval training on aerobic capacity in cardiac patients: a systematic review with meta-analysis. Biomed Res Int. 2017;2017:5420840.

12. Ramos J, Dalleck L, Tjonna A, Beetham K, Coombes J. The impact of highintensity interval training versus moderate-intensity continuous training on Vascular Function: a systematic review and meta-analysis. Sports Med (Auckland, NZ). 2015;45:679-92.

13. Weston K, Wisloff $\mathrm{U}$, Coombes J. High-intensity interval training in patients with lifestyle-induced cardiometabolic disease: a systematic review and meta analysis. Br J Sports Med. 2014;48:1227-34.

14. Vellers HL, Kleeberger SR, Lightfoot JT. Inter-individual variation in adaptations to endurance and resistance exercise training: genetic approaches towards understanding a complex phenotype. Mamm Genome. 2018;29(1-2):48-62.

15. Bouchard C, Sarzynski M, Rice TK, Kraus WE, Church TS, Sung YJ, et al. Genomic predictors of the maximal oxygen uptake response to standardized exercise training programs. J App Physiol. 2011;110:1160-70.

16. Williams CJ, Williams MG, Eynon N, Ashton KJ, Little JP, Wisloff U, et al. Genes to predict VO2max trainability: a systematic review. BMC Genomics. 2017;18(Suppl 8):831.

17. Defoor J, Vanhees L, Martens K, Matthijs G, Van Vlerken A, Zielinska D, et al. The CAREGENE study: ACE gene I/D polymorphism and effect of physical training on aerobic power in coronary artery disease. Heart. 2006;92(4):527-8.

18. Ghosh S, Vivar J, Sarzynski M, Sung Y, Timmons J, Bouchard C, et al. Integrative pathway analysis of a genome-wide association study of (V)O(2max) response to exercise training. J Appl Physiol. 2013;115(9):1343-59.

19. Rankinen T, Perusse L, Gagnon J, Chagnon Y, Leon A, Skinner J, et al. Angiotensin-converting enzyme ID polymorphism and fitness phenotype in the HERITAGE Family Study. J Appl Physiol. 2000;88(3):1029-35.

20. Rico-Sanz J, Rankinen T, Joanisse D, Leon A, Skinner J, Wilmore J, et al. Associations between cardiorespiratory responses to exercise and the C34T AMPD1 gene polymorphism in the HERITAGE Family Study. Physiol Genomics. 2003;14(2):161-6.

21. Rivera M, Dionne A, Fance T, Simoneau J, Perusse L, Chagnon M, et al. Muscle-specific creatine kinase gene polymorphism and $\mathrm{VO}_{2}$ max in the HERITAGE Family Study. Med Sci Sports Exerc. 1997;29(10):1311-7.

22. Rivera M, Perusse L, Simoneau J, Gagnon J, Dionne F, Leon A, et al. Linkage between a muscle-specific CK gene marker and VO2max in the HERITAGE Family Study. Med Sci Sports Exerc. 1999;31(5):698-701.

23. Thomaes T, Thomis M, Onkelinx S, Fagard R, Matthijs G, Buys R, et al. A genetic predisposition score for muscular endophenotypes predicts the increase in aerobic power after training: the CAREGENE study. BMC Genet. 2011;12:84.

24. Thompson PD, Tsongalis GJ, Seip RL, Biblie C, Miles M, Zoeller R, Visich P, Gordon P, Angelopoulos TJ, Pescatello L, Bausserman L, Moyna N. Apolipoprotein E genotype and changes in serum lipids and maximal oxygen uptake with exercise training. Metabolism. 2004;53(2):193-202.

25. Xu Y, Yang H, Ren Z, Yi L. Delta-aminolevulinate synthase 2 polymorphism is associated with maximal oxygen uptake after living-high exercisehigh training-low in a male Chinese population. Int J Clin Exp Med. 2015:8(11):21617-22.

26. Eynon N, Voisin S, Lucia A, Wang G, Pitsiladis Y. Preface: genomics and biology of exercise is undergoing a paradigm shift. BMC Genomics. 2017;18(8):825.

27. Sarzynski MA, Ghosh S, Bouchard C. Genomic and transcriptomic predictors of response levels to endurance exercise training. J Physiol. 2017;595(9):2931-9.

28. Bouchard C, Antunes-Correa LM, Ashley EA, Franklin N, Hwang PM, Mattsson CM, et al. Personalized preventive medicine: genetics and the response to regular exercise in preventive interventions. Prog Cardiovasc Dis. 2015;57(4):337-46.

29. Yan X, Eynon N, Papadimitriou ID, Kuang J, Munson F, Tirosh O, et al. The gene SMART study: method, study design, and preliminary findings. BMC Genomics. 2017;18(Suppl 8):821.
30. Pattyn N, Vanhees L, Cornelissen VA, Coekelberghs E, De Maeyer C, Goetschalckx K, et al. The long-term effects of a randomised trial comparing aerobic interval training versus continuous training in coronary artery disease: 1-year data from the SAINTEX-CAD study. Eur J Prev Cardiol. 2016;23(11):1154-64.

31. QIAsymphony. QIAsymphony DSP DNA Instructions for Use (Handbook). 2015. file:///C:/Users/camil/Downloads/HB-0977004_1069185_151035723_R4_HB_QS_DSP_DNA_Kit_0815_WW.pdf. Accessed 20 June 2019.

32. Taylor JL, Holland DJ, Keating SE, Leveritt MD, Gomersall SR, Rowlands AV, et al. Short-term and long-term feasibility, safety, and efficacy of highintensity interval training in cardiac rehabilitation: the FITR heart study randomized clinical trial. JAMA Cardiol. 2020;5:1382-9.

33. Stensvold D, Viken H, Steinshamn SL, Dalen H, Støylen A, Loennechen $J P$, et al. Effect of exercise training for five years on all cause mortality in older adults - the Generation 100 study: randomised controlled trial. BMJ. 2020;371:m3485.

34. Bonafiglia JT, Edgett BA, Baechler BL, Nelms MW, Simpson CA, Quadrilatero J, et al. Acute upregulation of PGC-1 a mRNA correlates with training-induced increases in SDH activity in human skeletal muscle. Appl Physiol Nutr Metab. 2017;42(6):656-66.

35. Bonafiglia JT, Rotundo MP, Whittall JP, Scribbans TD, Graham RB, Gurd BJ. Inter-individual variability in the adaptive responses to endurance and sprint interval training: a randomised crossover study. PloS One. 2016;11:e0167790.

36. Boyd CJ, Simpson CA, Jung ME, Gurd BJ. Reducing the intensity and volume of interval training diminishes cardiovascular adaptation but not mitochondrial biogenesis in overweight/obese men. PloS One. 2013;8(7):e68091.

37. Ma JK, Scribbans TD, Edgett BA, Boyd C, Simpson CA, Little JP, et al. Extremely low-volume, high intensity interval training improves exercise capacity and increases mitochondrial protein content in human skeletal muscle. Eur J Mol Integr Physiol. 2013;3(4).

38. Preobrazenski N, Bonafiglia JT, Nelms MW, Lu S, Robins L, LeBlanc C, et al. Does blood lactate predict the chronic adaptive response to training: a comparison of traditional and talk test prescription methods. Appl Physiol Nutr Metab. 2018.

39. Raleigh JP, Giles MD, Scribbans TD, Edgett BA, Sawula LJ, Bonafiglia $J$ T, et al. The impact of work-matched interval training on VO2peak and VO2 kinetics: diminishing returns with increasing intensity. Appl Physiol Nutr Metab. 2016;41(7):706-13.

40. Scribbans TD, Edgett BA, Vorobei K, Mltchell AS, Lioanisse SD, Matusiak $J B L$, et al. Fibre-specific response to endurance and low-volume high intensity interval training: striking similarities in acute and chronic adaptation. PloS One. 2014;9(6):e98119.

41. Forbes SC, Sletten N, Durrer C, Myette-Cote E, Candow D, Little JP. Creatine monohydrate supplementation does not augment fitness, performance, or body composition adaptations in response to four weeks of high-intensity interval training in young females. Int J Sport Nut Exer. 2017;27(3):285-92.

42. Francois M, Pistawka KJ, Halperin FA, Little JP. Cardiovascular benefits of combined interval training and post-exercise nutrition in type 2 diabetes. J Diabetes Complicat. 2017;32(2):226-33.

43. Ramos JS, Dalleck LC, Borrani F, Mallard AR, Clark B, Keating SE, et al. The effect of different volumes of high-intensity interval training on proinsulin in participants with the metabolic syndrome: randomised trial. Diabetologia. 2016;59(11):2308-20.

44. Genotek. Laboratory protocol for manual purification of DNA from $0.5 \mathrm{~mL}$ sample. 2019 https://www.dnagenotek.com/ROW/support/ protocols/preplT.html. Accessed 17 May 2019.

45. Univeristy of Oxford. Shellfish: Parallel PCA and data processing for genome-wide SNP data, UK. 2017. http://www.stats.ox.ac.uk/ davison/ software/shellfish/shellish.php. Accessed 17 May 2019.

46. COG-Genomics. PLINK 1.90 beta [v.1.90b3.36]. 2020. https://www.coggenomics.org/plink2. Accessed 10 Novr 2020.

47. Speed D, Balding DJ. MultiBLUP: improved SNP-based prediction for complex traits. GenomeR. 2014;24(9):1550-7.

48. Division of Biomedical Informatics Cincinnatit Children's Hospital Medical Center. ToppGene: a one-stop portal for gene list enrichment analysis and candidate gene prioritization based on functional annotations 
and protein interactions network, USA. 2019. https://toppgene.cchmc. org/. Accessed 13 Nov 2020.

49. Broad Insititue of MIT and Harvard. The GTEx Portal, USA \& UK. 2020. https://gtexportal.org/home/snp/rs73074755. Accessed 10 Nov 2020.

50. Yang J, Lee Hong S, Goddard ME, Visscher PM. GCTA: a tool for genome-wide complex trait analysis. Am J Hum Genet. 2011;7(88):76-82

51. Wu X, Hepner K, Castelino-Prabhu S, Do D, Kaye MB, Yuan XJ, et al. Evidence for regulation of the PTEN tumor suppressor by a membranelocalized multi-PDZ domain containing scaffold protein MAGI-2. Proc Natl Acad Sci USA. 2000;97(8):4233-8.

52. McKenzie J, Witkowski S, Ludlow A, Roth S, Hagberg J. AKT1 G205T genotypeinfluences obesity-related metabolic phenotypes and their responses to aerobic exercise training in older Caucasians. Exp Physiol. 2011;96(3):338-47.

53. Wisløff U, Bye A, Stølen T, Kemi OJ, Pollott GE, Pande M, et al. Blunted cardiomyocyte remodeling response in exercise-resistant rats. J Am Coll Cardiol. 2015;65(13):1378-80.

54. Sbroggiò M, Carnevale D, Bertero A, Cifelli G, De Blasio E, Mascio G, et al. IQGAP1 regulates ERK1/2 and AKT signalling in the heart and sustains functional remodelling upon pressure overload. Cardiovasc Res. 2011;91(3):456-64.

55. Bramswig NC, Bertoli-Avella AM, Albrecht B, Al Aqeel Al, Alhashem A, Al-Sannaa N, et al. Genetic variants in components of the NALCN-UNC80UNC79 ion channel complex cause a broad clinical phenotype (NALCN channelopathies). Hum Genet. 2018;137(9):753-68

56. Cochet-Bissuel M, Lory P, Monteil A. The sodium leak channel, NALCN, in health and disease. Front Cell Neurosci. 2014;8:132.

57. Park UH, Yoon SK, Park T, Kim EJ, Um SJ. Additional sex comb-like (ASXL) proteins 1 and 2 play opposite roles in adipogenesis via reciprocal regulation of peroxisome proliferator-activated receptor \{gamma\}. J Biol Chem. 2011;286(2):1354-63.

58. Erlich AT, Brownlee DM, Beyfuss K, Hood DA. Exercise induces TFEB expression and activity in skeletal muscle in a PGC-1a-dependent manner. Am J Physiol. 2018;314(1):C62-c72.

59. Mansueto G, Armani A, Viscomi C, D’Orsi L, De Cegli R, Polishchuk EV, et al. Transcription factor EB Controls Metabolic Flexibility During Exercise. Cell Metab. 2017;25(1):182-96

60. Theeuwes WF, Gosker HR, Schols A, Langen RCJ, Remels AHV. Regulation of PGC-1 a expression by a GSK-3B-TFEB signaling axis in skeletal muscle. Biochim Biophys Acta Mol Cell Res. 2020;1867(2):118610.

61. Andrade-Souza VA, Ghiarone T, Sansonio A, Santos Silva KA, Tomazini F, Arcoverde L, et al. Exercise twice-a-day potentiates markers of mitochondrial biogenesis in men. FASEB J. 2020;34(1):1602-19.

62. Petr M, Stastny P, Zajac A, Tufano JJ, Maciejewska-Skrendo A. The role of peroxisome proliferator-activated receptors and their transcriptional coactivators gene variations in human trainability: a systematic review. Int J Mol Sci. 2018;19(5):1472.

63. Yaghoob Nezhad F, Verbrugge SAJ, Schönfelder M, Becker L, Hrabě de Angelis M, Wackerhage H. Genes whose gain or loss-of-function increases endurance performance in mice: a systematic literature review. Front Physiol. 2019;10(262).

64. Stelzer G, Rosen N, Plaschkes I, Zimmerman S, Twik M, Fishilevich S, et al. The GeneCards Suite: from gene data mining to disease genome sequence analyses. Curr Protoc Bioinformatics. 2016;54(1).

65. Du K, Murakami S, Sun Y, Kilpatrick C, Luscher B. DHHC7 palmitoylates Glut4 and regulates Glut4 membrane translocation. J Biol Chem. 2017. https://doi.org/10.1074/jbc.M1 16.747139.

66. Serra-Juhé C, Martos-Moreno GÁ, de Pieri F, Flores R, González JR, Rodríguez-Santiago B, et al. Novel genes involved in severe early-onset obesity revealed by rare copy number and sequence variants. PLoS Genetics. 2017;13(5):e1006657.

67. Dickinson J, DiLugos A, Naymik M, Wolfe A, Curtis D, Huentelman M, Carroll C. Transcriptome response of human skeletal muscle to divergent exercise stimuli. JAP. 2018;124(6):1529-40.

68. Durkalec-Michalski K, Nowaczyk PM, Podgórski T, Kusy K, Osiński W, Jeszka J. Relationship between body composition and the level of aerobic and anaerobic capacity in highly trained male rowers. J Sports Med Phys Fitness. 2019;59(9):1526-35.

69. Radzimiński Ł, Szwarc A, Padrón-Cabo A, Jastrzębski Z. Correlations between body composition, aerobic capacity, speed and distance covered among professional soccer players during official matches. I Sports Med Phys Fitness. 2020;60(2):257-62.

70. Blanco-Gomez A, Castillo-Lluva S, Del Mar S-F, Hontecillas-Prieto L, Mao $\mathrm{JH}$, Castellanos-Martin A, et al. Missing heritability of complex diseases: enlightenment by genetic variants from intermediate phenotypes. BioEssays : News Rev Mol Cell Dev Biol. 2016;38(7):664-73.

71. Webborn N, Williams A, McNamee M, Bouchard C, Pitsiladis Y, Ahmetov I, et al. Direct-to-consumer genetic testing for predicting sports performance and talent identification: consensus statement. Br J Sports Med. 2015:49(23):1486-91.

72. Kikuchi N, Nakazato K. Effective utilization of genetic information for athletes and coaches: focus on ACTN3 R577X polymorphism. J Exerc Nutrition Biochem. 2015;19(3):157-64.

73. MacArther D, North K. Genes and human elite athletic performance. Hum Genet 2005:116:331-9.

74. Zeggini $\mathrm{E}$, loannidis J. Meta-analysis in genome-wide association studies. Pharmocogenomics. 2009;10(2):191-201.

75. Hoppeler H, Deciphering V. O2, max: limits of the genetic approach. J Exp Biol. 2018;221:21.

76. Pitsiladis YP, Tanaka M, Eynon N, Bouchard C, North KN, Williams AG, et al. Athlome Project Consortium: a concerted effort to discover genomic and other "omic" markers of athletic performance. Physiol Genomics. 2016;48(3):183-90.

\section{Publisher's Note}

Springer Nature remains neutral with regard to jurisdictional claims in published maps and institutional affiliations.

Ready to submit your research? Choose BMC and benefit from

- fast, convenient online submission

- thorough peer review by experienced researchers in your field

- rapid publication on acceptance

- support for research data, including large and complex data types

- gold Open Access which fosters wider collaboration and increased citations

- maximum visibility for your research: over $100 \mathrm{M}$ website views per year

At BMC, research is always in progress.

Learn more biomedcentral.com/submissions 